\title{
Paper Interleaving Technique Implementation to Reduce PAPR of OFDM Signal in Presence of Nonlinear Amplification with Memory Effects
}

\author{
Younes Aimer ${ }^{1,2}$, Boubakar Seddik Bouazza ${ }^{1}$, Smail Bachir $^{2}$, and Claude Duvanaud ${ }^{2}$ \\ ${ }^{1}$ Laboratory Technology of Communication, Faculty of Technology, University of Saida, Saida, Algeria \\ ${ }^{2}$ XLIM Laboratory UMR-CNRS 7252, Institute of Technology of Angoulême, University of Poitiers, Angoulême, France
}

\begin{abstract}
In OFDM systems, peak-to-average power ratio (PAPR) reduction of the signal is one of the main challenges that need to be overcome in order to use the transmitter in an efficient manner. As one of attractive techniques, interleaving can be used in PAPR reduction for multicarrier signals without spectrum distortion. In this paper, the authors propose to extend the possibilities of interleaving to improve PAPR reduction, to use a new coding of interleaver keys at the transmitter and a robust decoding procedure at the receiver. In order not to degrade the data rate, the use of null subcarriers to transmit side information to the receiver is proposed and evaluated.

Simulation results in the context of the WLAN 802.11a standard in the presence of a nonlinear power amplifier model with memory, show a reduction of PAPR of approximately $5.2 \mathrm{~dB}$, and an improvement of bit error rate and error vector magnitude of about 2 decades and $4 \%$ respectively, while respecting the spectral mask specification.
\end{abstract}

Keywords-downward compatibility, interleaving technique, interleaver key, OFDM systems, PAPR reduction.

\section{Introduction}

The next generations of wireless communication systems have to satisfy the customers' requirements in terms of quality and quantity of information. Because of its high spectral efficiency, robustness against interferences and frequency selective fading [1], as well as low complexity and implementation flexibility, Orthogonal Frequency Division Multiplexing (OFDM) has become the most common technique. For these reasons, OFDM is widely adopted as a physical layer (PHY) technology in recent wireless communication standards, such as Digital Audio/Video Broadcasting (DAB/DVB), Wireless Local Area Networks (WLAN), Wireless Metropolitan/Personal Area Networks (WMAN/WPAN), and WiMAX. OFDM is also included in the fifth generation $(5 \mathrm{G})$ cellular networks to support a high data transmission rate and to meet the required level of quality of service (QoS).
However, the major drawback of this technique is its high envelope fluctuation of the transmitted signal defined by the PAPR value [2]. Such a signal with high instantaneous values is degraded by the nonlinear characteristics of radiofrequency (RF) circuits, mainly the RF power amplifier (PA) [3]. To limit degradation, a back-off of the level is used, leading to the use of the PA with low power efficiency. Such a solution is costly, because it sacrifices the transmitter performance.

The objective of PAPR reduction methods is to limit these fluctuations to operate near the PA saturation region (high efficiency area), while maintaining the original data-rate and respecting such communication criteria as BER, EVM, downward compatibility ${ }^{1}$ and spectrum occupancy specifications.

PAPR reduction in OFDM systems has been a subject of intense research, based on which various techniques have been proposed in the literature. Among them, we can mention methods like clipping, clipping and filtering [4]- [6], active constellation extension (ACE) [7], [8], tone reservation (TR) [6], [9], [10], selected mapping (SLM) [11], [12], switching [13], coding methods [14] and partial transmit sequence (PTS) [8], [15], [16].

The authors in [17] proposed a PAPR reduction technique based on frequency interleaving of mapped symbols and the selection of the one that presents the lowest PAPR. However, the problem of the side information (SI) transmission is not addressed and only the principle of PAPR reduction is presented. In [18], a proposal to send SI on the chosen interleaver via pilots is made. This has the advantage of not affecting the data rate, but it influences the equalization and channel estimation at the receiver.

In this paper, we propose to use interleaving for PAPR reduction in WLAN 802.11a (Fig. 1) and to use null subcarriers (NS) available in the Wi-Fi-OFDM standard to transmit interleaver key-codes. The newly proposed technique

${ }^{1}$ Downward compatibility: additional signal processing at the transmitter do not need change at the receiver. 


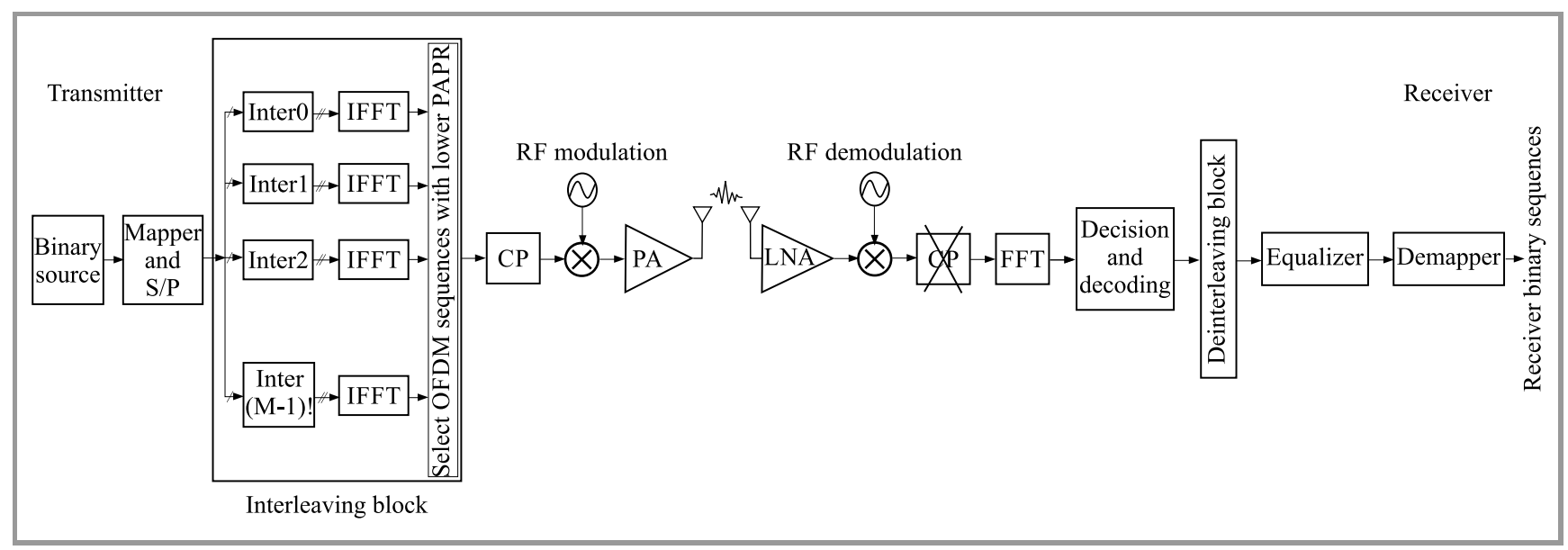

Fig. 1. Digital communication system with PAPR reduction based on frequency interleaving method.

provides a realistic solution by taking into account nonlinearity of RF-PA, without the need to transmit SI and respecting downward compatibility. The main idea is to test several interleaving possibilities, select the most efficient one and transmit its key-code on the minimum number of NS. A critical point of the proposed method is to ensure a correct transmission of the key-code despite degradation introduced by the transmission channel To ensure the quality level required, we propose a proper decoding process.

The proposed interleaving method is evaluated for WLAN 802.11a, in the presence of a PA model estimated from the measurements of a commercial PA, class $\mathrm{AB}$ with memory. Also, the key-code decoding process is evaluated and presents a low complexity and a high probability of error immunization against the AWGN channel. An effective scheme is proposed here, which constructs a favorable OFDM transmit signal with reduced PAPR.

Notation: Throughout this paper, small letters for vectors and matrices in the time-domain and capital letters for vectors and matrices in the frequency-domain are used.

\section{OFDM System}

\subsection{Problem Position}

In discrete time, the transmitted OFDM signals $x_{n}$ are obtained by taking Inverse Fast Fourier Transform (IFFT):

$$
x_{n}=\frac{1}{\sqrt{N}} \sum_{k=0}^{N-1} X_{k} \mathrm{e}^{\frac{\mathrm{j} 2 \pi n k}{N}}, \quad 0 \leq n \leq N-1,
$$

where $X_{k}, k=0,1, \ldots, N-1$ are input mapped symbols, usually modulated by a Quadrature Amplitude Modulation (QAM), and $k$ is the discrete-time index.

It is known that OFDM modulation is a particular case of multi-carrier transmission, where a single data stream is transmitted over a number of lower rate orthogonal subcarriers with the advantage of its high spectral distribution and efficiency, high data rate, robustness to multi-path channel and easier implementation. However, as shown in Eq. (1), the resulting signal is the superposition of $N$ independent narrow-band channels that can generate constructive and/or destructive sums, and consequently, high envelope fluctuations. These high instantaneous peaks limit the nonlinear RF-PA to operate at a lower average power efficiency, known as linear region, leading to a degradation of the transmitter efficiency. Improvement of power efficiency requires a reduction in fluctuations to maintain a higher average power without signal degradation. PAPR value quantifies this problem and defines the ratio between the maximum instantaneous power and average power for each OFDM symbol:

$$
\begin{aligned}
P A P R_{\mathrm{dB}} & =10 \cdot \log \frac{P_{\text {peak }}}{P_{\text {average }}} \\
P_{\text {peak }} & =10 \cdot \log \frac{\max _{0 \leq n \leq N-1}\left|x_{n}\right|^{2}}{\mathbb{E}\left|x_{n}\right|^{2}}
\end{aligned}
$$

where $P_{\text {peak }}$ represents the peak output power, $P_{\text {average }}$ means the average output power, $\mathbb{E}[\cdot]$ denotes the expected value operation and $x$ the vector of OFDM samples in timedomain such as $\mathbf{x}=\left[x_{0}, x_{1}, \ldots, x_{(N-1)}\right]^{T}$.

The complementary cumulative distribution function (CCDF) of PAPR is one of the most frequently used performance evaluation method for PAPR reduction techniques. It denotes the probability that PAPR of a data block exceeds a given threshold and is expressed as:

$$
C C D F=\mathbb{P}\left[P A P R>P A P R_{0}\right],
$$

where $\mathbb{P}[\cdot]$ is the probability function.

\section{Interleaving Method}

Interleaving is among the most commonly used techniques in digital communication systems, for its important benefits related to preventing the grouping of errors. Because coding is not capable of correcting a burst of errors, interleaving is used and consists in transforming these bursts 
into isolated errors, which makes their correction easier. The principle of interleaving is based on a simple breakdown of mapped symbols into more code words, before their transmission over the ODFM modulator. Thus, we define the number of code words $M$ (also called the depth of the interleaver) and the number of symbols per code word, called the word-length, and the denoted $K$. Figure 2 shows an example of an interleaver with $N=16$ subcarriers per OFDM symbol, $M=4$ code words and $K=4$ word-length. The mapped symbols are spread in a series over a matrix of $4 \times 4$ and sent-out in parallel, i.e. columnby-column, to the OFDM modulator. Note that we choose $N=16$ subcarriers to facilitate comprehension of the interleaving method.

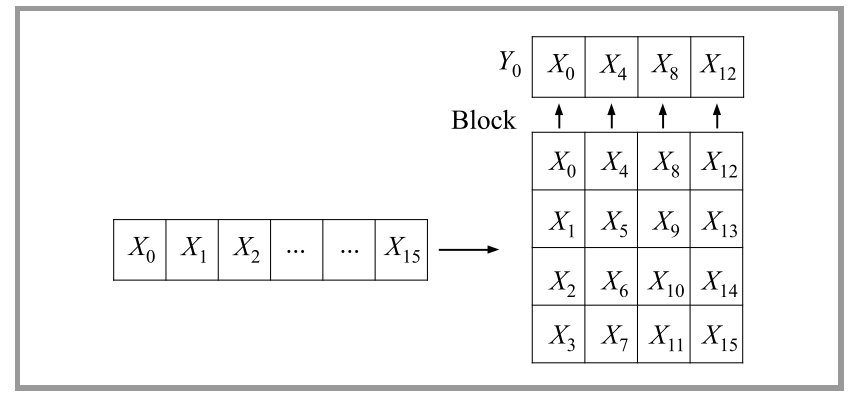

Fig. 2. Illustration of interleaving $(N=16, M=K=4)$.

Let us define the vector of modulated symbols such as:

$$
\mathbf{X}^{1 \times N}=\left[\begin{array}{lll}
X_{0} & X_{1} \cdots X_{N-1}
\end{array}\right] .
$$

To split this vector into $M$ code words, denoted as $Y_{f}$, we use the modulus function. If we denote the remainder after division of the code word position $f$ by $M$ as $s_{f}$, the code word $Y_{f}$ contains a group of mapped symbols which have the same remainder. We can describe the mathematical expression of the $f$-th codeword as follows:

$$
\mathbf{Y}_{f}^{K \times 1}=\left[\begin{array}{llll}
X_{M \cdot 0+s_{f}} & X_{M \cdot 1+s_{f}} \cdots X_{M \cdot(K-1)+s_{f}}
\end{array}\right]^{T} .
$$

At the receiver, there is no additional complexity to the system and only the inverse process is performed with the deinterleaver.

\subsection{Interleaving for PAPR Reduction}

The design of the interleaving method presented above is one of these proposed in the literature as a solution for reducing the risk of deformations of successive symbols in the same code word. In practice, only one kind of interleaving is performed as a function of the channel characteristics and the used coding. For PAPR reduction, the idea is to apply several kinds of interleaving at the transmitter. After IFFT operation, the smallest PAPR among them is chosen to be transmitted (Fig. 1). The major advantage of this technique is it its low complexity compared to the block coding techniques [14] or the PTS method [15], [16]. However, its major drawback is the need for the transmission of information about the used interleaver to carry out its decoding at the receiver, which degrades the data rate. Also, to insure the best reduction of the PAPR, a large number of interleaving possibilities is used, which increases the key length of each interleaver and degrades the data rate. In this work, we propose to deal with reducing PAPR using all possibilities of interleaving without the loss of in data rate, by:

- coding inter-key ${ }^{2}$ in within a minimum of length,

- transmitting used the inter-key via a minimum number of null subcarriers,

- and decoding the received inter-key with the use of a robust process.

\subsection{Interleaving Method Used}

In communication systems, adding redundancy in source coding and forward error correction (FEC) provides correlated data which influence the PAPR value. In [17], it is shown that the sequence of highly correlated binary data in multicarrier systems has a large PAPR. Thus, it is important to break down long correlation patterns to reduce high values of the complex envelope. In the proposed scheme (Fig. 3), we proposed to generate all $M$ ! possibilities (where

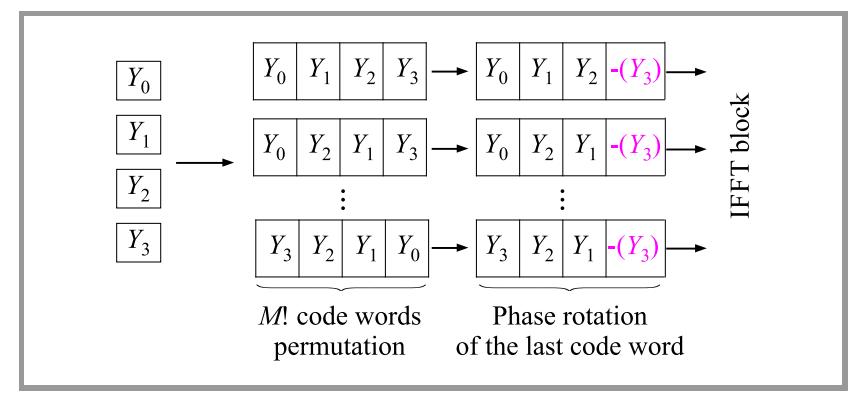

Fig. 3. Example of interleaving possibilities with $M=4$ code words.

(a)

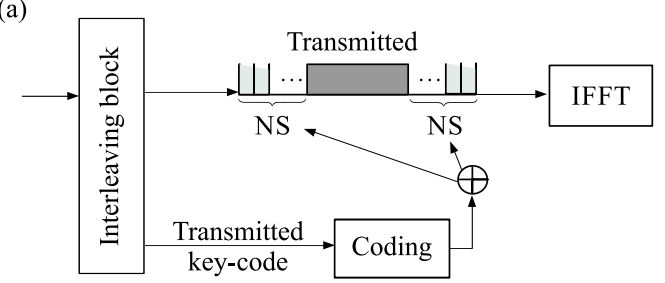

(b)

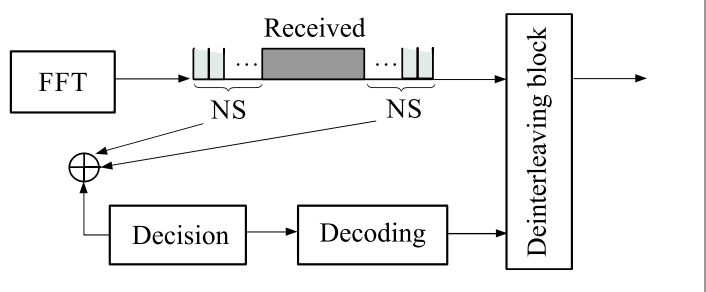

Fig. 4. Key-code on NS at the: (a) transmitter (b) receiver.

${ }^{2}$ inter-key: the code of the interleaver. 
$M$ is the number of code words) by permuting blocks in the new OFDM symbol and finally, reverse the last block. IFFT operation generates the time-domain signals and the sequence presenting the smallest PAPR is selected.

Each possibility of interleaving has its inter-key and the one chosen is transmitted to the receiver via null subcarriers (Fig. 4a). Here, the rank of the interleaver is coded in binary and mapped using quadrature amplitude modulation (QAM), by choosing the high-energy symbols of the constellation used.

Table 1 shows inter-keys in binary words of 6 bits and their equivalent in mapped symbols, where \pm 1 and $\pm \mathrm{j}$ indicate their position within the chosen 4-QAM constellation.

Table 1

Interleaver keys

\begin{tabular}{|c|c|c|c|}
\hline $\begin{array}{l}\text { Inter- } \\
\text { leaver }\end{array}$ & \multicolumn{2}{|r|}{ Binary code } & 4-QAM code \\
\hline 0 & {$[0$} & $\left.\begin{array}{lll}0,0 & 0,0 & 0\end{array}\right]$ & {$[-1-1 \mathrm{j},-1-1 \mathrm{j},-1-1 \mathrm{j}]$} \\
\hline 1 & {$[0$} & $\left.\begin{array}{lll}0,0 & 0,0 & 1\end{array}\right]$ & {$[-1-1 \mathrm{j},-1-1 \mathrm{j},-1+1 \mathrm{j}]$} \\
\hline$\vdots$ & \multicolumn{2}{|r|}{ : } & $\vdots$ \\
\hline 23 & & $\left.\begin{array}{lll}1,0 & 1,1 & 1\end{array}\right]$ & {$[-1+1 \mathrm{j},-1+1 \mathrm{j}, 1+1 \mathrm{j}]$} \\
\hline
\end{tabular}

\subsection{Inter-key Transmission via NS and Decoding Process}

In communication standards, one OFDM symbol is used to transmit data, pilots and also some reserved subcarriers

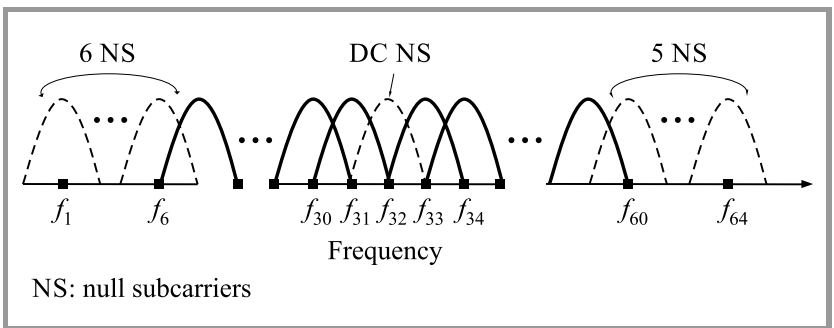

Fig. 5. OFDM symbol in WLAN 802.11a standard. which are set to zero (called null subcarriers). For example, in a Wi-Fi system, the OFDM symbol is presented in Fig. 5. Here, we propose to use these subcarriers in the interleaving method, where the inter-key of the optimal interleaver is transmitted with some of them. As a reminder, null subcarriers are used to prevent adjacent channel interference (ACI). In [19], [20] and [21] it has been shown for DVB-T, WiMAX and WLAN standards, respectively, that it results in a slight broadening of the spectrum but remains inside the spectrum mask.

\subsection{Inter-key Decoding Process}

Unfortunately and as for data subcarriers, PA nonlinearities and channel noise may affect the inter-key subcarriers transmitted, which will adversely affect the decoding process at the receiver.

To reduce the risk of error, we define a decoding process based on the comparison of the received inter-key with all possibilities using the XOR gate on each QAM position. The one that renders the maximum number of zeros is selected (Fig. 4b). The proposed decoding process is invoked for each OFDM symbol with the following operations:

\section{Variables:}

$\mathbf{C K}_{r}^{M \times 1}$ : received code-key,

$\mathbf{C K}^{M \times K}$ : matrix of all code-keys used at the transmitter,

$\mathbf{C K}_{c}^{M \times 1}$ : the correct code-key,

$\mathbf{R}^{M \times K}$ : resulting XOR operation matrix.

Step 1: extract $\mathrm{CK}_{r}^{M \times 1}$ from the received null-subcarriers and convert QAM symbols to binary.

Step 2: in $k$-th iteration, compare $\mathbf{C K}_{r}^{M \times 1}$ to each codekey row of matrix $\mathbf{C K}$ according to $\mathbf{R}[:, k]=\mathrm{CK}_{r} \oplus \mathbf{C K}[:, k]$, where $\mathbf{R}[:, k]$ denotes the $k$-th row of matrix $\mathbf{R}^{M \times K}$ and operation $\oplus$ is the binary XOR gate.

Step 3: Repeat step 2 for the chosen number $M$ of interleavers or stop when $\mathbf{R}[:, k]$ is a vector of zeros.

Step 4: $\mathbf{C K}_{c}$ corresponds to the line providing a maximum number of zeros.

Step 5: Select $\mathbf{C K}_{c}$ and deinterleave.

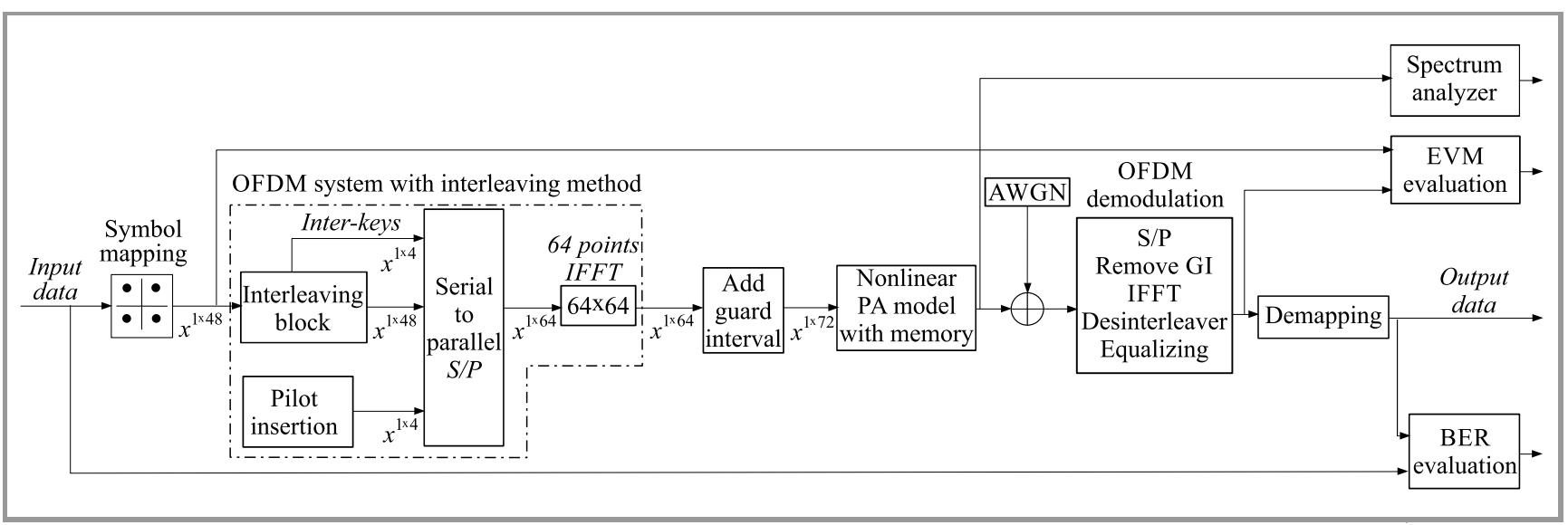

Fig. 6. Simulation block of Wi-Fi standard with interleaving method. 


\section{Simulation Results}

The proposed method is evaluated using the Matlab Mathworks platform for the WLAN 802.11a standard presented in Fig. 6. It adopts OFDM technique for its uplink (UP) and downlink (DL), with a frequency band of $5 \mathrm{GHz}$, and support data rate up to $54 \mathrm{Mbps}$ for an area coverage between user and transmitter of up to several meters.

Table 2

Simulation parameters

\begin{tabular}{|c|c|}
\hline Parameter & Value \\
\hline No. of sub-carriers & $\begin{array}{c}64 \text { (48 data, } \\
4 \text { pilots and } 12 \mathrm{NS} \text { ) }\end{array}$ \\
\hline Frequency band & $5 \mathrm{GHz}$ \\
\hline Bandwidth & $20 \mathrm{MHz}$ \\
\hline Modulation & 16-QAM \\
\hline No. of binary data & 4608000 bits \\
\hline No. of OFDM blocks & 2000 \\
\hline Performance metrics & CCDF, BER, spectrum \\
\hline $\begin{array}{c}\text { Num. of interleavers: } \\
\text { Case } 1\end{array}$ & $\begin{array}{c}4 !=24 \text { possibilities } \\
\text { coded on } 3 \mathrm{NS}\end{array}$ \\
\hline Case 2 & $\begin{array}{c}5 !=120 \text { possibilities } \\
\text { coded on } 4 \mathrm{NS}\end{array}$ \\
\hline
\end{tabular}

According to the parameters presented in Table 2, three contexts are studied:

- signals properties as a function of the number of interleaving possibilities,

- communication criteria (BER, EVM) using Gaussian channels,

- and frequency response in the presence of a nonlinear RF PA model.

Figure 7 presents a variation of the PAPR value as a function of the number of OFDM symbols for several interleav-

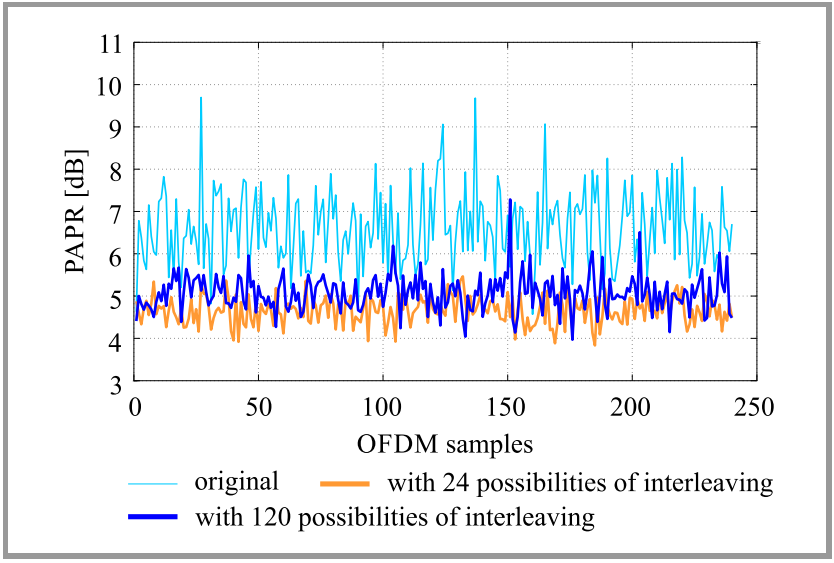

Fig. 7. PAPR in time-domain with and without interleaving. (For color pictures visit www.nit.eu/publications/journal-jtit)

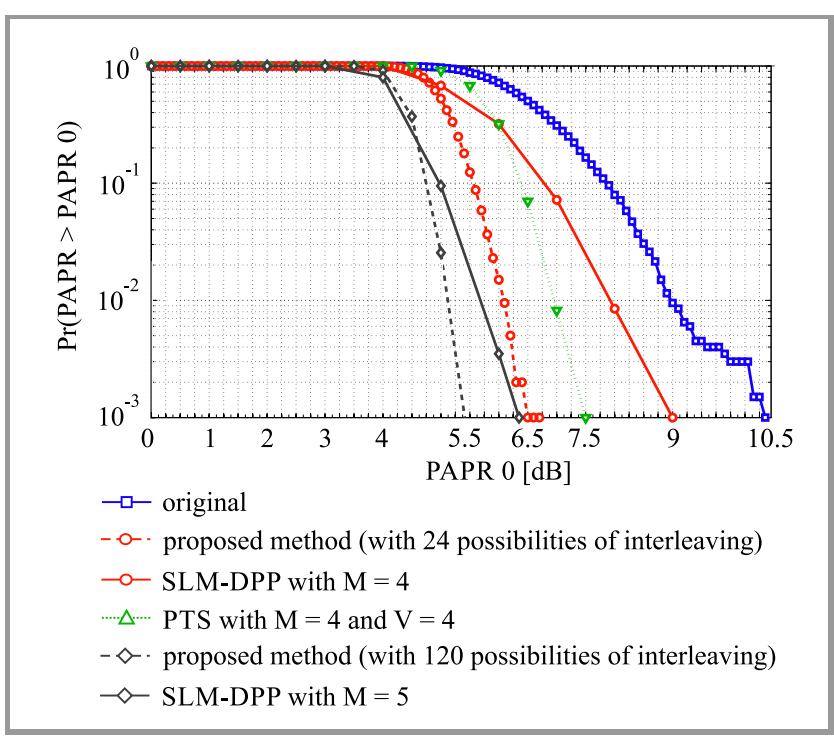

Fig. 8. Comparison of CCDF curves.

ing possibilities (24 and 120 possibilities of interleaving). We can see that, increasing number of possibilities offers better performance in terms of PAPR reduction.

Figure 8 shows a comparison (in terms of CCDF) of the proposed method with the PTS method presented in [16] and the SLM-DPP method given in [17]. The results obtained confirm those shown in Fig. 7, i.e., PAPR can be further reduced when we increase the number of interleaving possibilities.

For the probability of $10^{-3}$, it illustrates also a decrease in PAPR of up to $3.75 \mathrm{~dB}$ and $5.2 \mathrm{~dB}$ for 24 and 120 interleaving possibilities respectively, in comparison with the SLMDPP (for 4 and 5 blocks) and PTS method (for 4 blocks and 4 phases), which shows a reduction of $1.5 \mathrm{~dB}$ (SLMDPP for 4 blocks), $4 \mathrm{~dB}$ (SLM-DPP for 5 blocks) and $3 \mathrm{~dB}$, respectively.

\subsection{Nonlinear PA Model with Memory Effects}

To validate the solution, we perform several simulations in the presence of a nonlinear RF PA model.

Table 3

Model parameters

\begin{tabular}{|c|c|}
\hline & Values \\
\hline \hline$b_{0,1}$ & $-3,1+\mathrm{j} 2.93$ \\
\hline$b_{1,1}$ & $-2,86 \cdot 10^{-1}+\mathrm{j} 2.19 \cdot 10^{-1}$ \\
\hline$b_{0,3}$ & $5.15 \cdot 10^{-2}-\mathrm{j} 4.2 \cdot 10^{-2}$ \\
\hline$b_{1,3}$ & $7.58 \cdot 10^{-3}-\mathrm{j} 4.34 \cdot 10^{-3}$ \\
\hline$b_{0,5}$ & $-3.49 \cdot 10^{-4}+\mathrm{j} 2.75 \cdot 10^{-4}$ \\
\hline$b_{1,5}$ & $-4.93 \cdot 10^{-5}+\mathrm{j} 1.37 \cdot 10^{-5}$ \\
\hline
\end{tabular}

We consider a model with memory of a commercial class AB power amplifier (SZP-2026Z from RF Micro Devices) 
designed for WLAN 802.11 equipment, where its input to output relationship, in baseband format, is expressed as [21]:

$$
y(t)=\sum_{i=0}^{m} \sum_{p=0}^{P} b_{i, 2 p+1} \cdot|u(t-i)|^{2 p} \cdot u(t-i),
$$

where $P$ is the nonlinearity order and $m$ the memory depth. For $P=2$ and $m=1$, parameters $b_{i, 2 p+1}$ are estimated using a multicarrier signal with a bandwidth of $20 \mathrm{MHz}$. Table 3 summarizes the model parameters.

In Fig. 9 we have plotted the AM/AM static characteristic presented by the output signal power depending on the input signal power, which describes the SZP-2026Z power amplifier. The $1 \mathrm{~dB}$ compression point corresponding to $\mathrm{IBO}=0 \mathrm{~dB}$, is achieved for input power equal to $20 \mathrm{dBm}$.

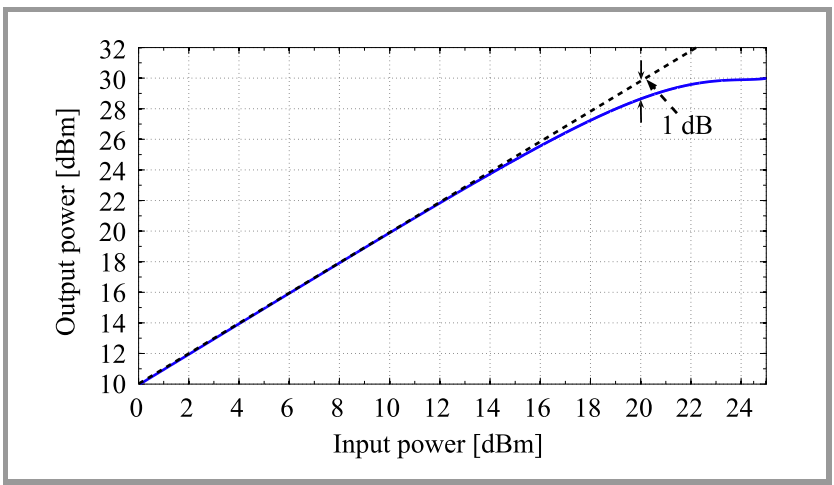

Fig. 9. AM/AM static characteristics of the SZP-2026Z amplifier.

To see the effect of the power amplifier used on the $\mathrm{Wi}$ Fi-OFDM signal with application of the reduction method, we have plotted the AM/AM dynamic characteristic with and without the interleaving method (Fig. 10).

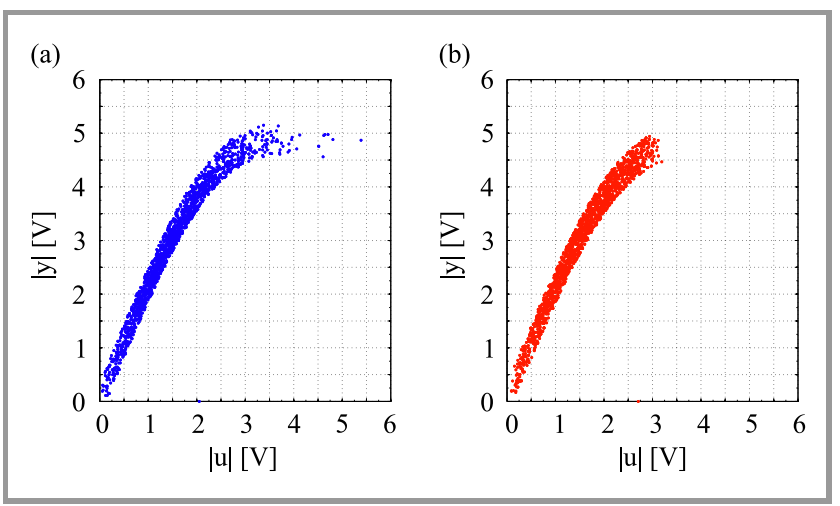

Fig. 10. AM/AM dynamic characteristics without (a) and with (b) interleaving method.

After comparison of the two figures, we note that the application of the interleaving method reduces the signal dynamic and then the nonlinear distortion generated by the amplifier.

To evaluate the proposed system we estimate its sensibility to channel noise in the presence of a power amplifier.

Figure 11 presents EVM of the received signal versus SNR for $\mathrm{IBO}=-2 \mathrm{~dB}$, with and without interleaving (for 24 and

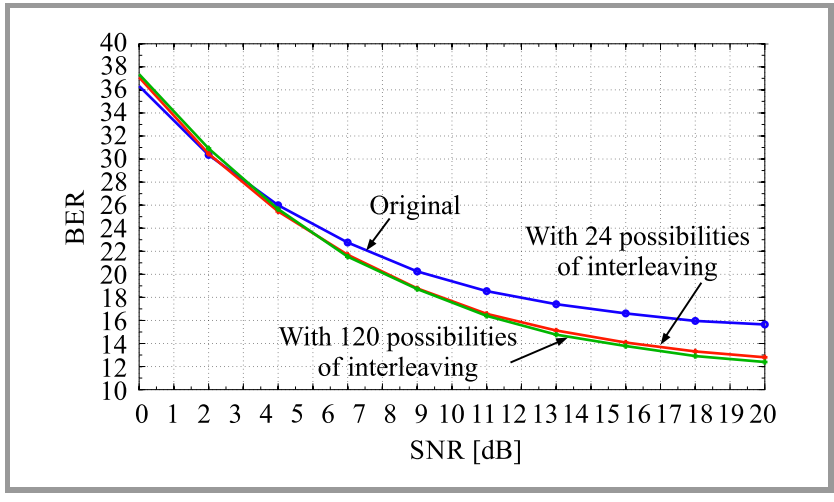

Fig. 11. EVM versus $\mathrm{SNR}$ for Gaussian channels ( $\mathrm{IBO}=-2 \mathrm{~dB}$ ).

120 possibilities of interleaving) for a Gaussian channel and in the presence of a nonlinear power amplifier.

From these results, one can observe that for a low SNR value $(\mathrm{SNR}<6 \mathrm{~dB})$, the curves with and without the interleaving method behave almost in the same manner. At this SNR level, the noise power to be added is important. Consequently, the impact of channel noise is preponderant compared to the nonlinearity of the amplifier, and that prevents improvement in EVM.

On the other hand, at higher SNR values (SNR $>12 \mathrm{~dB})$, the impact of noise is negligible compared to the nonlinearity of the amplifier. In this case the interleaving method allows a good improvement in the EVM result.

In the same context, Fig. 12 presents BER results versus $\mathrm{SNR}$ for an $\mathrm{IBO}=-2 \mathrm{~dB}$, where we can observe that the use of the proposed method offers an improvement in BER, with high SNR values (SNR $>12 \mathrm{~dB})$.

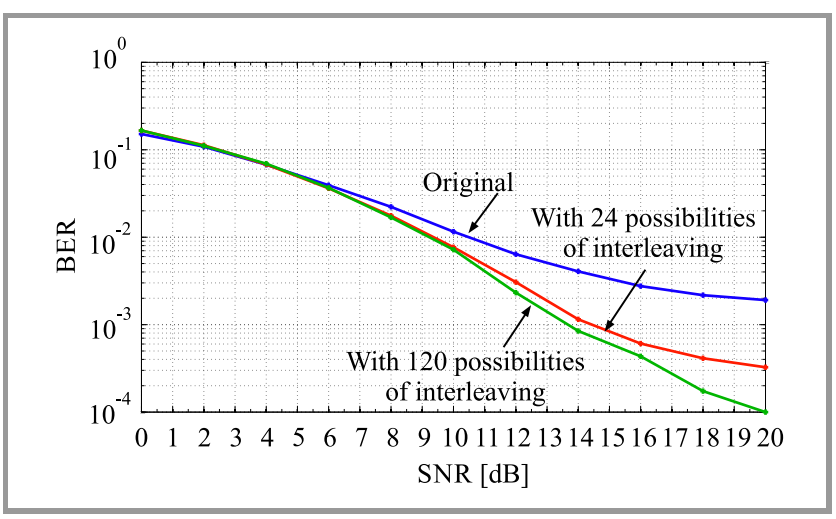

Fig. 12. BER versus $S N R$ for Gaussian channels $(I B O=-2 \mathrm{~dB})$.

Figures 13 and 14 present the comparison of EVM and BER curves versus $\mathrm{IBO}$, for $\mathrm{SNR}=16 \mathrm{~dB}$. These results confirm the suitability of the interleaving method proposed, as it allows for an improvement in EVM of about $2.5 \%$ and $3 \%$, and in BER with a factor of one decade, one decade and a half for 24 and 120 possibilities of interleaving, respectively.

Based on the above and with the same transmission quality (identical EVM or identical BER), the interleaving method makes it possible to obtain a gain of $2 \mathrm{~dB}$ in IBO. In other words, by applying the interleaving method, the am- 


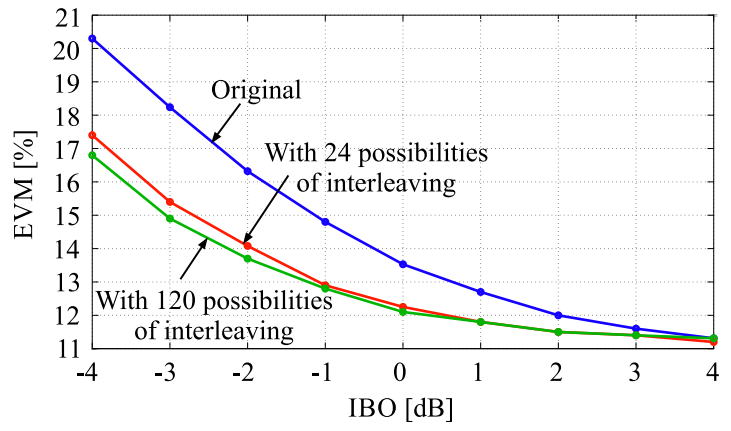

Fig. 13. $E V M$ curves vs. $\mathrm{IBO}(\mathrm{SNR}=16 \mathrm{~dB})$.

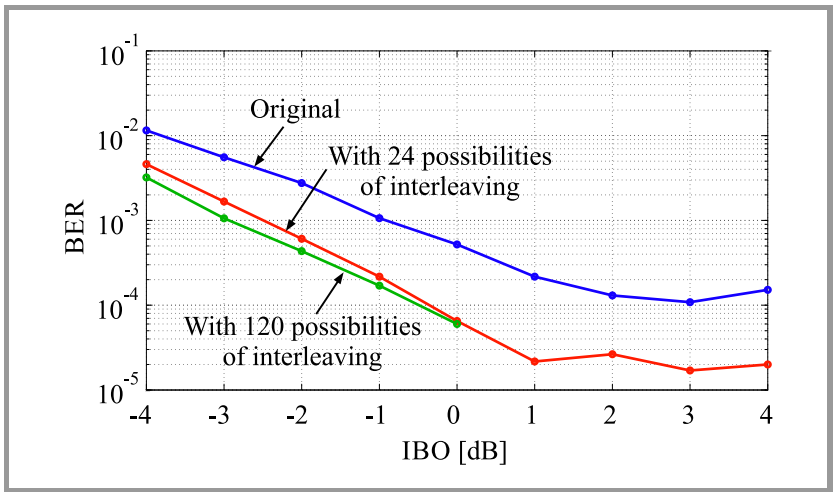

Fig. 14. BER curves vs. $\mathrm{IBO}(\mathrm{SNR}=16 \mathrm{~dB})$.

plifier can be used with an increase of $2 \mathrm{~dB}$ of the input power level, and with a higher power efficiency compared to the scenario without the method being applied, for the same QoS.

Figure 15 shows a constellation, with (see Fig. 15a) and without the interleaving method (see Fig. 15b) at the amplifier output for an IBO equal to $-2 \mathrm{~dB}$. Application of the interleaving method makes it possible to reduce the dispersion on the constellation points.

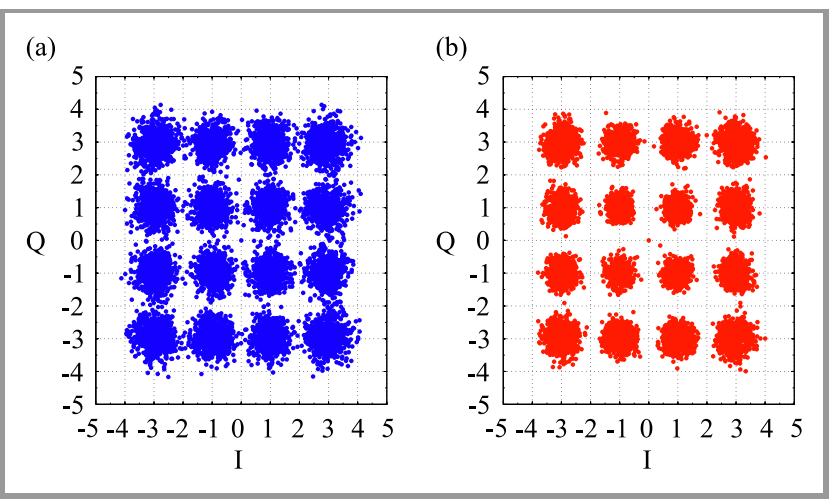

Fig. 15. Constellation at the amplifier output without (a), and with (b) the application of the interleaving method $(\mathrm{IBO}=-2 \mathrm{~dB})$.

In Fig. 16 the impact of NS is shown for Wi-Fi-OFDM signal at an $\mathrm{IBO}=-2 \mathrm{~dB}$, before and after application of the interleaving method (for 24 and 120 possibilities of interleaving).

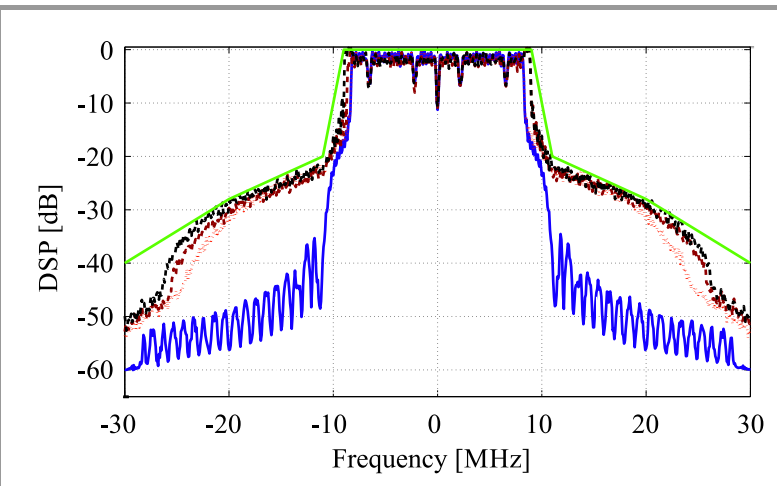

— original шишиши original with PA

- - - with 24 possibilities of interleaving and PA

- - with 120 possibilities of interleaving and PA

Fig. 16. Impact of nonlinear PA model with memory on frequency responses with and without interleaving $(\mathrm{IB}=-2 \mathrm{~dB})$.

As expected, the use of null subcarriers affects the spectrum with a larger bandwidth and spurious component in the adjacent bands due to nonlinear effects. However, they will remain still under the mask specification of the standard used.

ACPR values are calculated after the amplifier for different IBO levels, with the lower and upper bandwidth of $25 \mathrm{MHz}$ for each, with and without the interleaving method. The results are shown in Table 4. In terms of ACPR, they are very close, both without and with the interleaving method for 24 and 120 possibilities.

\section{Conclusions}

In this paper, an original implementation of the interleaving method is proposed for PAPR reduction and is evaluated in terms of its robustness, against a nonlinear PA and noisy channel. The principle is based on the selection of the interleaving possibility with the lowest PAPR. The main objective of the method is to avoid optimization processes, such as those applied in TR [21].

In practice, we proposed a coding process where the selected interleaver is coded with an inter-key. In order not to degrade the data rate, the interleaver is transmitted to the receiver via a minimum number of null subcarriers. Such a technique ensures downward compatibility and offers a PAPR improvement of about $5.2 \mathrm{~dB}$ over the original signal.

In order to find the correct interleaver at the receiver, despite the transmission errors, a practical decoding process has been presented. A nonlinear power amplifier with memory was inserted to evaluate the proposed method in terms of QoS and energy efficiency. From these results, we observed an improvement in EVM of about $3 \%$ and an improvement in BER by a factor of more than one decade. So, for a given quality in terms of EVM or BER, the interleaving method enables to use the power amplifier at a higher IBO $(-2 \mathrm{~dB})$ rate, leading to greater power efficiency values. 
Table 4

ACPR Results

\begin{tabular}{|c|c|c|c|c|c|c|}
\hline \multirow{2}{*}{} & \multicolumn{6}{|c|}{ Adjacent Channel Power Ratio [dB] } \\
\cline { 2 - 7 } & \multicolumn{2}{|c|}{ Original } & With 24 possibility & \multicolumn{2}{c|}{ With 120 possibility } \\
\cline { 2 - 7 } & Lower & Upper & Lower & Upper & Lower & Upper \\
\hline \hline $\mathrm{IBO}=-4 \mathrm{~dB}$ & -22.13 & -22.00 & -21.83 & -21.74 & -21.78 & -21.75 \\
\hline $\mathrm{IBO}=-2 \mathrm{~dB}$ & -25.09 & -24.94 & -24.88 & -24.77 & -24.26 & -24.20 \\
\hline $\mathrm{IBO}=0 \mathrm{~dB}$ & -28.39 & -28.21 & -28.12 & -28.00 & -27.25 & -27.10 \\
\hline $\mathrm{IBO}=2 \mathrm{~dB}$ & -31.53 & -31.32 & -31.03 & -30.92 & -30.00 & -30.00 \\
\hline $\mathrm{IBO}=4 \mathrm{~dB}$ & -34.04 & -33.88 & -33.22 & -33.12 & -32.00 & -31.90 \\
\hline
\end{tabular}

The power amplifier output spectrum demonstrates the possibilities offered in terms of performance improvement, while observing WLAN 802.11a specifications. The use of null subcarriers for transmitting SI is therefore not an obstacle to the generalization of the interleaving method and its application to next generation of wireless communication systems.

\section{Acknowledgments}

Research reported in this publication is supported by PHCTassili project of the Hubert Curien Partnership.

\section{References}

[1] M.-O. Pun, M. Morelli, and C.-C. J. Kuo, Multi-carrier Techniques for Broadband Wireless Communications: A Signal Processing Perspective, Communication and Signal Processing (Book 3). London: Imperial College Press, 2007 (ISBN: 978-1860949463).

[2] R. Prasad, OFDM for Wireless Communications Systems. Norwood, MA: Artech House, 2004 (ISBN: 978-1580537964).

[3] T. Jiang and Y. Wu, "An Overview: Peak-to-Average Power Ratio Reduction Techniques for OFDM Signals", IEEE Transact. on Broadcast., vol. 54, no. 2, pp. 257-268, 2008 (doi: 10.1109/TBC.2008.915770)

[4] D. Guel and J. Palicot, "Clipping Formulated As an Adding Signal Technique for OFDM Peak Power Reduction", in Proc. VTC Spring 2009 - IEEE 69th Vehicul. Technol. Conf., Barcelona, Catalonia, Spain, 2009, pp. 1-5 (doi: 10.1109/VETECS.2009.5073442).

[5] R. Saroj, "A Cooperative Additional Hybrid and Clipping technique for PAPR reduction in OFDM System", in Proc. 2016 Fifth Int. Conf. on Eco-friendly Comput. and Commun. Sys. (ICECCS), Bhopal, Madhya Pradesh, India, 2016, pp. 53-57 (doi: 10.1109/Eco-friendly.2016.7893241).

[6] M. J. Azizipour and K. Mohamed-pour, "Clipping noise estimation in uniform tone reservation scenario using OMP algorithm", in Proc. 2016 8th Int. Symp. on Telecommun. (IST), Teheran, IRI, 2016, pp. 500-505 (doi: 10.1109/ISTEL.2016.7881871).

[7] Z. Zheng and G. Li, "An Efficient FPGA Design and Performance Testing of the ACE Algorithm for PAPR Reduction in DVB-T2 Systems", IEEE Transact. on Broadcast., vol. 63, no. 1, pp. 134-143, 2017 (doi: 10.1109/TBC.2016.2593401).

[8] R. Saroj, "Performance evaluation of hybrid ACE-PTS PAPR reduction techniques", in Proc. 2016 11th Int. Conf. on Computer Engineer. Sys. (ICCES), Cairo, Egypt, 2016, pp. 407-413 (doi: 10.1109/ICCES.2016.7822039).
[9] J. Tellado-Mourelo, "Peak to Average Power Ratio Reduction for Multicarrier Modulation", PhD thesis, Stanford University, 1999.

[10] H. Sohtsinda, S. Bachir, C. Perrine, and C. Duvanaud, "An evaluation of hybrid tone reservation method for PAPR reduction using power amplifier with memory effects", IEEE Int. Conf. on Electron. Circuits and Sys. (ICECS), Monte Carlo, 2016, pp. 676-679 (doi: 10.1109/ICECS.2016.7841292).

[11] R. W. Bauml, R. G. H. Fischer, and J. B. Huber, "Reducing the peak-to-average power ratio of multicarrier modulation by selected mapping”, Electron. Let., vol. 32, no. 22, pp. 2056-2057, 1996 (doi: 10.1049/el:19961384).

[12] J. Ji, G. Ren, and H. Zhang, "A Semi-Blind SLM Scheme for PAPR Reduction in OFDM Systems With Low-Complexity Transceiver", IEEE Transact. on Vehicul. Technol., vol. 64, no. 6, pp. 2698-2703, 2015 (doi: 10.1109/TVT.2014.2345262).

[13] M. S. Hossain and T. Shimamura, "Low-complexity null subcarrierassisted OFDM PAPR reduction with improved BER", IEEE Commun. Let., vol. 20, no. 11, pp. 2249-2252, 2016 (doi: 10.1109/LCOMM.2016.2598801).

[14] A. E. Jones, T. A. Wilkinson, and S. K. Barton, "Block coding scheme for reduction of peak to mean envelope power ratio of multicarrier transmission schemes", Electron. Let., vol. 30, no. 25, pp. 2098-2099, 1994, (doi: 10.1049/el:19941423).

[15] S. H. Muller and J. B. Huber, "OFDM with reduced peak-to-average power ratio by optimum combination of partial transmit sequences", Electron. Let., vol. 33, no. 5, pp. 368-369, 1997 (doi: 10.1049/el:19970266).

[16] A. Hanprasitkum, A. Numsomran, P. Boonsrimuang, and P. Boonsrimuang, "Improved PTS method with new weighting factor technique for FBMC-OQAM systems", in Proc. 19th Int. Conf. on Adv. Communic. Technol. (ICACT), Bongpyeong, South Korea, 2017, pp. 143-147 (doi: 10.23919/ICACT.2017.7890073).

[17] J. H. Wen, G. R. Lee, C. C. Kung, and C. Y. Yang, "Coding schemes applied to peak-to-average power ratio (PAPR) reduction in OFDM systems", in Proc. 2008 Int. Wirel. Communic. and Mob. Comput. Conf., Crete Island, Greece, 2008, pp. 807-812 (doi: 10.1109/IWCMC.2008.139)

[18] H.-G. Ryu, S.-K. Kim, and S.-B. Ryu, "Interleaving method without side information for the PAPR reduction of OFDM system", in Proc. Int. Symp. on Communic. and Inform. Technol., Sydney, NSW, Australia, 2007, pp. 72-76 (doi: 10.1109/ISCIT.2007.4391987).

[19] I. M. Mahafeno, Y. Louet, and J.-F. Helard, "Peak-to-average power ratio reduction using second order cone programming based tone reservation for terrestrial digital video broadcasting systems', IET Communic., vol. 3, no. 7, pp. 1250-1261, 2009 (doi: 10.1049/iet-com.2008.0372).

[20] S. Hu, G. Wu, Q. Wen, Y. Xiao, and S. Li, "Nonlinearity reduction by tone reservation with null subcarriers for WiMAX system", Wireless Person. Communic., vol. 54, no. 2, pp. 289-305, 2010 (doi: 10.1007/s11277-009-9726-z). 
[21] B. Koussa, S. Bachir, C. Perrine, C. Duvanaud, and R. Vauzelle, "A comparison of several gradient based optimization algorithms for PAPR reduction in OFDM systems", in Proc. 2nd International Conference on Communications, Computing and Control Applications CCCA'12, Marseilles, France, 2012, pp. 1-6 (doi: 10.1109/CCCA.2012.6417865).

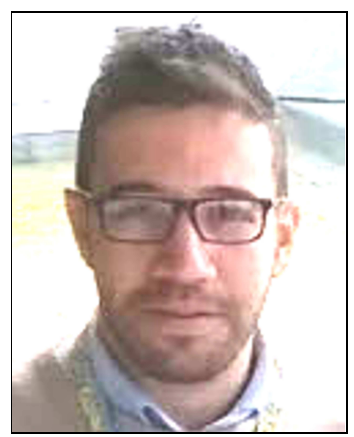

Younes Aimer received his B.Sc. and M.Sc. in Electronics and Telecommunications from the University of Saida, Algeria in 2011 and 2013, respectively. Since 2014 he has been a Ph.D. student in Signal Processing and Telecommunications at the Laboratory of Technologies and Communications, University of Saida, Algeria, and since 2016 at the XLIM laboratory, Department of Smart Networks, University of Poitiers, France in co-tutelle (in: electronics, microelectronics, nanoelectronics and microwaves). His current research interests are in digital communications, wireless and mobile communications and signal processing. E-mail: aimer.younes@univ-saida.dz

Laboratory Technology of Communication

Faculty of Technology

University of Saida

BP 138 En-Nasr

20000 Saida, Algeria.

E-mail: younes.aimer@univ-poitiers.fr

XLIM Laboratory UMR-CNRS 7252

Institute of Technology of Angoulême

University of Poitiers

4 avenue de Varsovie

16021 Angoulême cedex, France

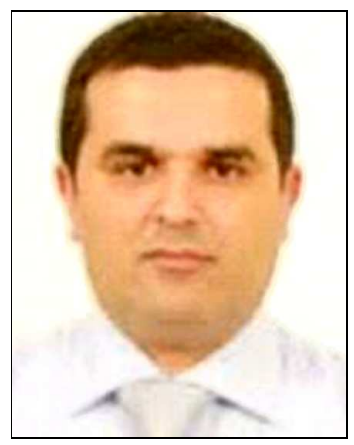

Boubakar Seddik Bouazza received his B.Sc., M.Sc. and Ph.D. degrees, all in electrical engineering, from University of Sidi Bel Abbes, Algeria, in 1996, 1999 and 2006, respectively. In 1999 he joined the University of Saida (Algeria) as an assistant professor and he is a lecturer at the Electronics Department since 2006. Since 2012, he is a director of the communications technology laboratory. He held visiting positions at École Nationale Supérieure de Télécommunications de Bretagne (ENST), Brest, France, as a Ph.D student. His current research in- terests are in digital communications, wireless and mobile communications, channel coding, turbo codes and bandwidth-efficient coded modulation schemes.

E-mail: boubakar.bouazza@univ-saida.dz

Laboratory Technology of Communication

Faculty of Technology

University of Saida

BP 138 En-Nasr

20000 Saida, Algeria

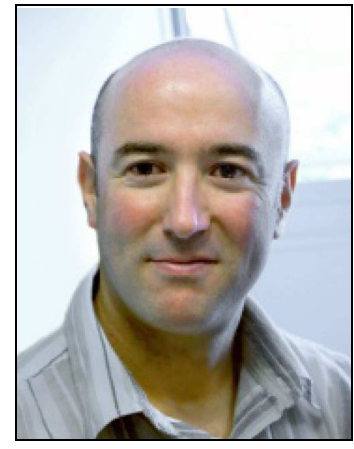

Smail Bachir received both B.Sc. and M.Sc. in Signal Theory from Polytechnic School of Algeria in 1997. He joined the scientific department of Leroy Somer Society and the University of Poitiers in France where he received his Ph.D. degree in Automatic and Electrical Engineering in 2002 . He is presently an Associate Professor at the University of Poitiers and a researcher at XLIM laboratory with the Department of Smart Networks and Systems. His research interests include signal processing, parameter identification, electronic devices and wireless circuits.

E-mail: smail.bachir@univ-poitiers.fr

XLIM Laboratory UMR-CNRS 7252

Institute of Technology of Angoulême

University of Poitiers

4 avenue de Varsovie

16021 Angoulême cedex, France

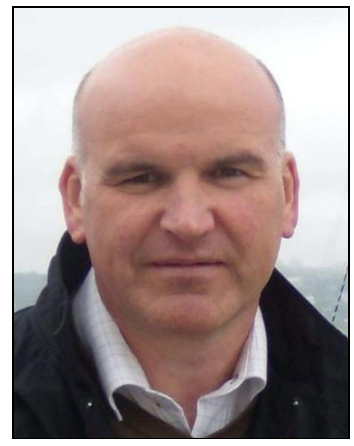

Claude Duvanaud received his Ph.D. in Electronics and Communication Engineering from the University of Limoges, France, in 1993 and the a habilitation degree (Diriger des Recherches) from the University of Poitiers in 2003. He currently is an Associate Professor at the University Institute of Technology, Angoulême, University of Poitiers, France. His research interests include modeling, simulation and design of nonlinear power amplifiers and communication systems.

E-mail: claude.duvanaud@univ-poitiers.fr

XLIM Laboratory UMR-CNRS 7252

Institute of Technology of Angoulême

University of Poitiers

4 avenue de Varsovie

16021 Angoulême cedex, France 